\title{
Ters Veri Zarflama Analizi Yöntemiyle Global 2000 Firmalarında Kaynak Tahsisi
}

\author{
Süleyman ÇAKIR \\ Recep Tayyip Erdoğan Üniversitesi, İ̈BF, İşletme Bölümü, \\ suleyman.cakir@erdogan.edu.tr, ORCID:0000-0003-0334-8777
}

Öz:

Ters Veri Zarflama Analizi (VZA) modelleri, bir grup karar birimi arasından belirlenen bir karar biriminin bazı veya tüm girdi/çıktı düzeyleri değiştirildiğinde, etkinlik skoru sabit kalmak şartıyla, bu karar biriminin diğer girdi/çıktılarının optimal miktarlarını hesaplamayı amaçlayan modellerdir. Bu çalışmada önerilen Ters VZA modeliyle "Forbes" dergisinin düzenlediği "Global 2000" listesinin 2019 yılı sıralamasında yer alan 21 adet havayolu firması için kaynak tahsisi yapılmıștır. Uygulama sonuçları önerilen modelin organizasyonlarda kaynakların yeniden tahsisi amacıyla kullanılabilecek pratik bir yöntem olduğu ve karar vericiler için sağlam ve yararlı bilgiler ürettiğini göstermektedir.

Anahtar kelimeler: Ters Veri Zarflama Analizi, Kaynak Tahsisi, Havayolu Firmaları JEL Sinıflandırma Kodları: C61, M11

\section{Resource Allocation in Global 2000 Companies using Inverse Data Envelopment Analysis ${ }^{1}$}

\begin{abstract}
Inverse Data Envelopment Analysis (DEA) models desire to calculate optimal input/output levels of a specific Decision Making Unit when some or all of its input/output levels are altered, while retaining the efficiency score. In this paper, using the proposed inverse DEA model, resource allocation has been conducted for 21 airline companies of the "Global 2000" list for the year 2019 organized by "Forbes" magazine. Application results show that the suggested model is a practical method that can be used for the re-allocation of resources in organizations and produces robust and useful information for decision makers.
\end{abstract}

Keywords: Inverse Data Envelopment Analysis, Resource Allocation, Airlines Companies JEL Classification Codes: C61, M11

\footnotetext{
${ }^{1}$ Extended abstract is presented at the end of the article.

Geliş Tarihi (Received): 06.02.2020 - Kabul Edilme Tarihi (Accepted): 18.05.2020

Atıfta bulunmak için/Cite this paper:

Çakır, S. (2020) Ters veri zarflama analizi yöntemiyle Global 2000 firmalarında kaynak tahsisi. Çankırı Karatekin Üniversitesi IIBBF Dergisi, 10 (1), 305-318. Doi: 10.18074/ckuiibfd.685775.
} 


\section{Giriş}

Charnes, Cooper ve Rhodes (1978) tarafından geliştirilen Veri Zarflama Analizi (VZA) aynı sektörde faaliyet gösteren ve benzer girdiler kullanıp benzer çıktılar üreten karar verme birimleri (KVB)'nin göreli etkinliğini ölçen parametrik olmayan bir optimizasyon tekniğidir. Günümüze kadar hemen her tür organizasyonun performans ölçümü amacıyla yararlanılan VZA'nın kapsamlı bir literatür taraması için Emrouznejad ve Yang (2018) çalışmasına başvurulabilir.

VZA girdi ve çıktı değişkenlerindeki değişikliklere karşı oldukça duyarlı bir yöntemdir. Öyle ki, verilerde meydana gelecek bir küçük bir değişim bile etkin üretim sınırının ve dolayısıyla KVB'lerin etkinlik skorlarının değişmesine neden olabilir (Lertworasirikul, Charnsethikul ve Fang 2011). Bu noktada ortaya çıkan önemli bir problem ise, kısa dönemde girdi-çıktı setindeki değişimler karşısında karar biriminin etkinlik skorunun nasıl korunacağı konusudur. Literatürde kaynakların yeniden tahsisi (resource re-allocation) olarak da bilinen bu tür problemler ters optimizasyon (inverse optimization) problemleri olarak ele alınmakta ve verili bir optimal çözümden hareketle sistem parametreleri yeniden hesaplanmaktadir.

Literatürdeki ilk Ters VZA modeli Wei, Zhang ve Zhang (2000) tarafından geliştirilmiştir. Ters VZA modelleri bir grup KVB arasından bir karar biriminin girdi (çıktı) gözlemleri değiş̧tiğinde o karar biriminin etkinlik skoru sabit kalacak şekilde çıktı (girdi) değişkenlerinde ne miktarda bir değişim gerektiğini hesaplamaktadır. Daha sonra ters VZA problemi çok amaçlı doğrusal programlama (ÇADP) modeline dönüştürülüp çözümlenmektedir. Wei vd. (2000) çalışmasından sonra literatürde bu modelin genişletilmiş ve modifiye edilmiş versiyonları önerilmiştir. Söz konusu çalışmalar aşağıda belirtilmektedir.

Yan, We ve Hao (2002), Wei vd. (2000) modelini karar vericilerin özel tercihlerini ve politikalarını dâhil edebilecekleri şekilde ek kısıtlar içeren yeni bir modele genişletmişlerdir. Jahanshahloo, Hosseinzadeh ve Moradi (2004) değerlendirmeye alınan karar biriminin etkinlik skoru sabit kalacak veya iyileşecek şekilde o karar biriminin bazı veya tüm çıktıları artırıldığında girdi değişkenlerinin ne kadar artırılması gerektiğini hesaplayan ters VZA modelleri önermişlerdir. Hadi-Vencheh ve Foroughi (2006) diğer ters VZA modellerinden farklı olarak karar birimlerinin bazı girdi (çıktı) değişkenlerindeki artış ve azalışlardan dolayı diğer çıktı (girdi) değişkenlerinde meydana gelen artış ve azalışları eşzamanlı olarak hesaplamayı mümkün kılan bir genelleştirilmiş ters VZA modeli önermiştir. Sözkonusu çalışmada ayrıca, Wei vd. (2000) tarafindan geliştirilen modelin bazı durumlarda istenmeyen sonuçlar verdiği ispatlanmıştır. Abdollah, Ali ve Majid (2008) tarafından zayıf etkinlik yerine güçlü etkinlik modeline dayalı olan ve çıktılardaki artış karşısında girdilerdeki artışları hesaplayan bir ters VZA modeli geliştirilmiştir. Lertworasirikul vd. (2011) ölçeğe göre değişken getiri (ÖGDG) varsayımına dayalı bir ters VZA modeli önermiştir. 
Ghiyasi (2015), Lertworasirikul vd. (2011) tarafindan önerilen modelde bazı matematiksel hatalar bulunduğunu iddia ederek bu hataların düzeltildiği bir VZA modeli ortaya koymuşlardır. Jahanshahloo, Hosseinzadeh, Rostamy-Malkhalifeh ve Ghobadi (2014) Russell modeline dayalı olarak geliştirdikleri ters VZA modeliyle Pareto optimal şekilde girdi/çıktı hesaplaması gerçekleştirmişlerdir. Zhang ve Cui (2016) ters VZA modelleriyle çözümlenebilecek farklı senaryoları analiz etmiş ve girdi ve çıktıya yönelik farklı VZA modelleri sunmuşlardır. Literatürdeki mevcut VZA modellerinin kesin sayılara dayalı olması ve gerçek hayat problemlerinin belirsizlik ve bulanıklık içermesinden hareketle HadiVencheh, Hatami-Marbini, Ghelej ve Gholami (2014) aralık sayılara dayalı bir aralık ters VZA modeli geliştirmiştir. Aralık sayılar karar alma sürecinin içerdiği belirsizliği pratik bir şekilde temsil edebilmekte ve klasik modellere kıyasla problem hakkında daha az bilgiye gereksinim duymaktadır.

Yukarıda bahsedilen ters VZA modellerinin literatürdeki kullanım alanlarına örnek olarak; Çin devletinin 2015-2024 yılları arasındaki sürdürülebilir gelişimi için yatırım probleminin ele alındığı (Chen, Wang, Lai ve Feng, 2017), Çin bankalarının operasyonel etkinliğinin hesaplandığı $(\mathrm{Yu}$, Shao, You, Wu, ve Xu 2019), imalat firmalarının sera gazı emisyon kotalarının yeniden tahsis probleminin çözümlendiği (Emrouznejad, Yang ve Amin, 2019; Wegener ve Amin, 2019), yeni ürün geliştirmede hedef belirleme amacıyla kullanıldığ (Lim, 2016); eyaletlerin enerji etkinliğinin ölçüldüğü (Ghiyasi, 2017), otomobil imalat firmasının satış sonrası müşteri hizmetlerinin performansının ölçüldüğü (Farzipoor Saen ve Seyedi Hosseini Nia, 2019), Tunus'taki tarım alanlarının sulama amaçlı su talebini tahmin etmek amacıyla kullanıldığı (Frija, Wossink, Buysse, Speelman ve Huylenbroeck, 2011) çalışmalar gösterilebilir.

Bu çalışmada Hadi-Vencheh ve Foroughi (2006) tarafından literatüre kazandırılan ters VZA modeliyle "Forbes" dergisinin açıkladığı "Global 2000" listesinde yer alan 21 adet havayolu firması için etkinlik analizi yapılmıştır. Literatürde ters VZA modeliyle etkinlik ölçümü yapılan az sayıda çalışma bulunması nedeniyle bu çalışmanın literatüre katkı sunacağı düşünülmektedir. Çalışmanın bundan sonraki kısımları aşağıdaki biçimde organize edilmiştir. İkinci kısımda uygulamada kullanılan ters VZA modeliyle ilgili bilgi verilmiştir. Uygulamanın yer aldığı üçüncü bölümü sonuç kısmı takip etmektedir.

\section{Yöntem}

Çalışmanın bu kısmında klasik ve ters VZA modelleri hakkında bilgi verilmiştir. İlk olarak klasik VZA modelleri açıklanmıştır. 


\subsection{VZA Yöntemi}

Etkinliği ölçülecek $\mathrm{n}$ adet karar biriminin $m$ adet girdi değişkeni kullanarak $s$ adet çıktı değişkeni ürettiği varsayılsın. Sözkonusu $\mathrm{KVB}_{\mathrm{j}}{ }^{\prime}$ lerin $(\mathrm{j}=1, \ldots, n)$ girdileri $\mathrm{x}_{\mathrm{ij}}$ $(\mathrm{i}=1, \ldots, \mathrm{m})$ ve çıktıları $\mathrm{y}_{\mathrm{rj}}(\mathrm{r}=1, \ldots, \mathrm{s})$ ile gösterilsin. Herhangi bir karar birimi olan $\mathrm{KVB}_{\mathrm{k}}$ 'nın diğer karar birimlerine göre etkinliğini hesaplamak için ölçeğe göre sabit getiri (ÖGSG) varsayımı altında Charnes vd. (1978) tarafından geliştirilen girdi yönlü klasik CCR-VZA modeli denklem (1) ile gösterilmektedir.

$$
\begin{array}{ll}
\sum_{\mathrm{j}=1}^{\operatorname{Min} \theta_{\mathrm{k}}} & \\
\sum_{\mathrm{j}=1}^{\mathrm{n}} \lambda_{\mathrm{j}} \mathrm{y}_{\mathrm{rj}} \geq \mathrm{y}_{\mathrm{rk}} & \mathrm{i}=1,2, \ldots, \mathrm{m} \\
\lambda_{\mathrm{j}} \geq 0 & \mathrm{j}=1,2, \ldots, \mathrm{s} \\
&
\end{array}
$$

Yukarıda gösterilen CCR modeline dışbükeylik kısıtı olan $\sum_{j=1}^{n} \lambda_{j}=1$ eklenmesiyle ÖGDG altında etkinlik ölçümü yapmayı sağlayan girdi yönlü BCC-VZA (Banker, Charnes ve Cooper, 1984) modeli elde edilmektedir.

Girdi yönelimli VZA modelleri belirli bir çıktı bileşimine ulaşmayı sağlayacak minimum girdi düzeylerini hesaplamayı amaçlamaktadır. Çıktı yönlü modeller ise belirli bir girdi bileşimi ile üretilebilecek maksimum çıktı düzeylerini hesaplamaktadır. Çıktıya yönelik BCC-VZA modeli ise eşitlik (2) ile gösterildiği gibidir.

$$
\begin{array}{lr}
\sum_{j=1}^{\operatorname{Mak} \varphi_{\mathrm{k}}} \lambda_{\mathrm{j}} \mathrm{x}_{\mathrm{ij}} \leq \mathrm{x}_{\mathrm{ik}} & \mathrm{i}=1,2, \ldots, \mathrm{m} \\
\sum_{\mathrm{j}=1}^{\mathrm{n}} \lambda_{\mathrm{j}} \mathrm{y}_{\mathrm{rj}} \geq \varphi \mathrm{y}_{\mathrm{rk}} & \mathrm{r}=1,2, \ldots, \mathrm{s} \\
\sum_{\mathrm{j}=1}^{\mathrm{n}} \lambda_{\mathrm{j}}=1 & \\
\lambda_{\mathrm{j}} \geq 0 & \mathrm{j}=1,2, \ldots, \mathrm{n}
\end{array}
$$

Tanım. Eğer Model (1) ve (2)'nin optimal değerleri 1 çıkarsa $\left(\theta_{\mathrm{k}}, \varphi_{\mathrm{k}}=1\right)$ her iki modelde de $\mathrm{KVB}_{\mathrm{k}}$ etkin bir karar birimi kabul edilmektedir. Diğer durumlarda karar birimleri için etkinsiz yorumu yapılmaktadır. 


\subsection{Ters VZA Modelleri}

Ters VZA modelleri organizasyonların girdi ve çıktı faktörlerinde meydana gelebilecek kısa dönemli değişiklikler karşısında kaynakların yeniden optimal şekilde tahsisi konusunda karar vericilere yardımcı olmayı amaçlayan VZA modelleridir. Örneğin, bir firmanın etkinlik skoru sabit kalacak şekilde ürettiği çıktılarının miktarında değişiklik yapılmak istenmektedir. $\mathrm{Bu}$ durumda bu firmanın girdileri ne miktarda değiştirilmelidir? Problemi çözmek için, $\mathrm{KVB}_{\mathrm{k}}$ 'nın çıktılarının $\mathrm{y}_{\mathrm{k}}$ düzeyinden $\beta_{\mathrm{k}}=\mathrm{y}_{\mathrm{k}}+\Delta \mathrm{y}_{\mathrm{k}}$ düzeyine değiştirildiği varsayılsın. Burada, $\Delta \mathrm{y}_{\mathrm{k}} \in \mathfrak{R}^{\mathrm{s}}$ olmaktadır. $\mathrm{Bu}$ durumda $\mathrm{KVB}_{\mathrm{k}}$ 'nın etkinlik skorunu değiştirmeyecek girdi vektörü $\alpha_{\mathrm{k}}$ miktarları hesaplanacaktır. Bu amaçla aşağıda gösterilen ÇADP modelinden yararlanılabilir (Hadi-Venchech, 2006):

$$
\begin{array}{ll}
\sum_{j=1}^{\mathrm{Min}}\left(\alpha_{1}, \alpha_{2,}, \ldots, \alpha_{\mathrm{m}}\right) & \\
\sum_{\mathrm{j}=1}^{\mathrm{n}} \lambda_{\mathrm{j}} \mathrm{y}_{\mathrm{rj}} \geq \beta_{\mathrm{ij}} \leq \theta_{\mathrm{k}}^{*} \alpha_{\mathrm{k}} & \mathrm{i}=1,2, \ldots, \mathrm{m} \\
\lambda_{\mathrm{j}} \geq 0 & \mathrm{r}=1,2, \ldots, \mathrm{s} \\
& \mathrm{j}=1,2, \ldots, \mathrm{n}
\end{array}
$$

Burada, $\alpha=\mathrm{x}_{\mathrm{k}}+\Delta \mathrm{x}, \quad \Delta \mathrm{x} \in \mathfrak{R}^{\mathrm{m}} \quad \mathrm{KVB}_{\mathrm{k}}{ }^{\prime}$ nın etkinlik skorunun değişmemesini sağlayacak olan girdi miktarlarını göstermektedir. Denklem (3) ile gösterilen ÇADP modelinin çözümü için Steuer (1986) tarafından önerilen ağırlıklı toplam yöntemi (weighted sum method) kullanılabilir. Önerilen yöntem basit hesaplama prosedürüne sahip olduğu ve karar vericilerin tercihlerini amaç fonksiyonunda dikkate alabildiği için literatürde sıklıkla tercih edilmektedir. Sözkonusu ağırlıklı model denklem (3)’e uygulandığında aşağıdaki biçimi almaktadır.

$$
\begin{array}{ll}
\operatorname{Min} \sum_{\mathrm{i}=1}^{\mathrm{m}} \mathrm{w}_{\mathrm{i}} \alpha_{\mathrm{i}} & \\
\sum_{\mathrm{j}=1}^{\mathrm{n}} \lambda_{\mathrm{j}} \mathrm{x}_{\mathrm{ij}} \leq \theta_{\mathrm{k}}^{*} \alpha_{\mathrm{k}} & \mathrm{i}=1,2, \ldots, \mathrm{m} \\
\sum_{\mathrm{j}=1}^{\mathrm{n}} \lambda_{\mathrm{j}} \mathrm{y}_{\mathrm{rj}} \geq \beta_{\mathrm{k}} & \mathrm{r}=1,2, \ldots, \mathrm{s} \\
\lambda_{\mathrm{j}} \geq 0 & \mathrm{j}=1,2, \ldots, \mathrm{n}
\end{array}
$$

Buradaki $w_{i}, \quad(i=1, \ldots, m)$ karar vericilerin i. amaç fonksiyonuna atadığı ağırlığ sembolize etmektedir. Bu çalışmada basitlik için tüm amaç fonksiyonlarına " 1 " değeri atanmıştır. 
Benzer şekilde, $\mathrm{KVB}_{\mathrm{k}}{ }^{\prime}$ 'nın girdi hacmini $\mathrm{x}_{\mathrm{k}}$ düzeyinden $\alpha_{\mathrm{k}}=\mathrm{x}_{\mathrm{k}}+\Delta \mathrm{x}, \Delta \mathrm{x} \in \mathfrak{R}^{\mathrm{m}}$ düzeyine çıkarmak istediği varsayılsın. Etkinlik skoru $\varphi_{\mathrm{k}}$ sabit kalması için bu karar birimi hangi düzeyde çıtı üretmelidir? Bahsedilen optimizasyon probleminin çözümü için eşitlik (5) ile gösterilen ÇADP modelinden yararlanılmaktadir.

$$
\begin{array}{ll}
\text { Mak } \sum_{r=1}^{\mathrm{s}} \mathrm{w}_{\mathrm{r}} \beta_{\mathrm{r}} & \\
\sum_{\mathrm{j}=1}^{\mathrm{n}} \lambda_{\mathrm{j}} \mathrm{x}_{\mathrm{ij}} \leq \alpha_{\mathrm{k}} & \mathrm{i}=1,2, \ldots, \mathrm{m} \\
\sum_{\mathrm{j}=1}^{\mathrm{n}} \lambda_{\mathrm{j}} \mathrm{y}_{\mathrm{rj}} \geq \varphi_{\mathrm{k}}^{*} \beta_{\mathrm{k}} & \mathrm{r}=1,2, \ldots, \mathrm{s} \\
\lambda_{\mathrm{j}} \geq 0 & \mathrm{j}=1,2, \ldots, \mathrm{n}
\end{array}
$$

ÖGSG varsayımına dayalı olan Model (4) ve (5)'e dışbükeylik kısıtı $\sum \lambda_{j}=1$ eklenerek BCC modelleri için de benzer hesaplamalar yapılabilir.

\section{Uygulama}

$\mathrm{Bu}$ çalışmada "Forbes" dergisinin geleneksel olarak her yıl açıkladığı "Global 2000" listesinin 2019 yılı sıralamasında yer alan 21 adet havayolu firması için ters VZA uygulaması yapılmıştır. Forbes dergisi dünyanın en büyük 2000 fïmasını satışlar, kâr, aktifler ve pazar değeri büyüklüklerinin ağırlıklı ortalamalarını dikkate alarak belirlemektedir. 61 ülkenin halka açı (public) firmalarının yer aldığ 2019 yılı listesinde ABD 575 firma ile başı çekmektedir. Çin ve Hong Kong 309'ar, Japonya ise 223 firma ile ABD'yi takip etmektedir. Çalışmada kullanılan veriler Forbes dergisinin web sitesinden (https://www.forbes.com/global2000/list/ erişim Tarihi: 10 Ocak 2019) derlenmiştir. Uygulama kapsamındaki havayolu firmaları Tablo 1'de gösterilmektedir.

Tablo 1: Uygulama Kapsamındaki Havayolu Firmaları

\begin{tabular}{llll}
\hline Kodu & Adı & Kodu & Adı \\
\hline F1 & Delta Air Lines & F12 & Air France-KLM \\
F2 & United Continental Holdings & F13 & Türk Hava Yollar1 \\
F3 & American Airlines Group & F14 & Singapore Airlines \\
F4 & Southwest Airlines & F15 & Qantas Airways \\
F5 & Deutsche Lufthansa & F16 & Cathay Pacific Airways \\
F6 & International Airlines & F17 & Hainan Airlines \\
F7 & All Nippon Airways & F18 & Air Canada \\
F8 & China Southern Airlines & F19 & Latam Airlines \\
F9 & China Eastern Airlines & F20 & Alaska Air Group \\
F10 & Japan Airlines & F21 & EasyJet \\
F11 & Ryanair Holdings & & \\
\hline
\end{tabular}


Uygulamada kullanılacak girdi ve çıktı değişkenleri ilgili literatür ve veri kısıtı dikkate alınarak seçilmiştir. Söz konusu değişkenler Tablo 2'de gösterilmektedir.

Tablo 2: VZA Uygulamasında Kullanılan Değişkenler

\begin{tabular}{|c|c|c|}
\hline Değişken & Türü & Açıklama \\
\hline Çalışan sayısı & Girdi & 2019 yılında firmadaki toplam çalışan sayısı \\
\hline Aktif toplamı & Girdi & 2019 y1lında firmanın aktif toplamı \\
\hline Piyasa değeri & Çıktı & 2019 yılında firmanın toplam piyasa değeri \\
\hline Aktif karlılı̆̆ & Çıktı & $\begin{array}{l}2019 \text { yılında firmanın net karının toplam varlıklarına } \\
\text { oranı }\end{array}$ \\
\hline Net kâr marji & Cikt1 & 2019 yılında firmanın net karının net satıșlarına oranı \\
\hline
\end{tabular}

Buna göre uygulamada kullanılan veri seti Tablo 3'te sunulmuştur.

Tablo 3: Uygulamada Kullanılan Veri Seti

\begin{tabular}{llllll}
\hline & $\begin{array}{l}\text { Çalışan Sayıs1 } \\
\text { (kişi) }\end{array}$ & $\begin{array}{l}\text { Aktif Toplam1 } \\
\text { (milyar \$) }\end{array}$ & $\begin{array}{l}\text { Piyasa Değeri } \\
(\text { milyar \$) }\end{array}$ & $\begin{array}{l}\text { Aktif Karlılığ1 } \\
(\%)\end{array}$ & $\begin{array}{l}\text { Net Kâr Marj1 } \\
(\%)\end{array}$ \\
\hline F1 & 89000 & 61,8 & 38,1 & 0,066 & 0,091 \\
F2 & 92000 & 50,6 & 23,3 & 0,045 & 0,055 \\
F3 & 128900 & 60,6 & 15,4 & 0,023 & 0,031 \\
F4 & 58800 & 27,3 & 28,5 & 0,092 & 0,114 \\
F5 & 135534 & 44,4 & 12,1 & 0,059 & 0,061 \\
F6 & 63531 & 32 & 14,4 & 0,106 & 0,118 \\
F7 & 43466 & 23,6 & 12 & 0,038 & 0,048 \\
F8 & 100831 & 36 & 14,8 & 0,013 & 0,021 \\
F9 & 77005 & 34,5 & 14,9 & 0,012 & 0,025 \\
F10 & 33038 & 17,6 & 11,8 & 0,068 & 0,090 \\
F11 & 14853 & 13,6 & 16,1 & 0,096 & 0,143 \\
F12 & 81527 & 33,2 & 5,3 & 0,015 & 0,015 \\
F13 & 35205 & 20,5 & 3,4 & 0,041 & 0,064 \\
F14 & 24350 & 20,2 & 8,6 & 0,024 & 0,041 \\
F15 & 30248 & 13,1 & 6,5 & 0,050 & 0,051 \\
F16 & 26623 & 24,3 & 7 & 0,012 & 0,021 \\
F17 & 24772 & 27,3 & 6,7 & 0,007 & 0,020 \\
F18 & 28300 & 14,1 & 6,6 & 0,009 & 0,009 \\
F19 & 41170 & 17,6 & 6,5 & 0,011 & 0,019 \\
F20 & 23376 & 11,5 & 7,6 & 0,038 & 0,053 \\
F21 & 14000 & 9,1 & 6,3 & 0,066 & 0,091 \\
\hline
\end{tabular}


Havayolu firmaları için öncelikle klasik VZA modelleriyle etkinlik ölçümü gerçekleştirilmiştir. Girdi ve çıtı yönlü BCC ve CCR modelleriyle hesaplanan etkinlik skorları Tablo 4'te gösterilmiştir.

Tablo 4: Havayolu Firmalarma Ait CCR-VZA ve BCC-VZA Etkinlik Skorları

\begin{tabular}{llll}
\hline KVB & CCR Skoru & BCC-I Skoru & BCC-O Skoru \\
\hline F1 & 0,521 & 1,000 & 1,000 \\
F2 & 0,389 & 0,438 & 1,501 \\
F3 & 0,215 & 0,219 & 2,452 \\
F4 & 0,882 & 1,000 & 1,000 \\
F5 & 0,230 & 0,265 & 1,704 \\
F6 & 0,472 & 1,000 & 1,000 \\
F7 & 0,430 & 0,496 & 2,018 \\
F8 & 0,347 & 0,361 & 2,089 \\
F9 & 0,365 & 0,378 & 2,047 \\
F10 & 0,566 & 0,661 & 1,422 \\
F11 & 1,000 & 1,000 & 1,000 \\
F12 & 0,135 & 0,274 & 5,687 \\
F13 & 0,298 & 0,453 & 2,227 \\
F14 & 0,360 & 0,583 & 2,200 \\
F15 & 0,539 & 0,702 & 1,831 \\
F16 & 0,243 & 0,528 & 2,799 \\
F17 & 0,250 & 0,567 & 2,842 \\
F18 & 0,395 & 0,655 & 2,508 \\
F19 & 0,312 & 0,522 & 3,034 \\
F20 & 0,558 & 0,843 & 1,517 \\
F21 & 0,826 & 1,000 & 1,000 \\
\hline Ortalama & 0,444 & 0,616 & 2,094 \\
\hline
\end{tabular}

Buna göre CCR modeline göre sadece F11 (Ryanair Holdings) firması etkin çıkmıştır. 21 havayolu işletmesinin ortalama etkinlik skoru ise 0,444 gibi düşük bir düzeyde gerçekleşmiştir. Girdi yönlü BCC (BCC-I) ve çıktı yönlü BCC (BCCO) modellerine göre ise etkinlik skorları 1 çıkan F1, F4, F6, F11 ve F21 firmaları etkin olarak değerlendirilmektedir. Ryanair Holdings firması (F11) her üç modelde de etkin çıkan tek firma olmuştur. Genel bir değerlendirme yapılacak olursa, havayolu firmalarının kaynaklarını etkin kullanamadıkları söylenebilir.

CCR modelinde etkin çıkan Ryanair Holdings firması için ters VZA modeliyle yeniden kaynak tahsisi yapılmak istendiği varsayılsın. Bu firmanın piyasa değeri, aktif karlılığı ve net kar marjını ifade eden çıktı vektörü $y_{1}=(16,1 ; 0.0956$; $0.1429)$ 'ten $\beta_{1}=(25 ; 0.15 ; 0.1)$ şekline dönüştürülmek istenmektedir. Başka bir deyişle, firmanın piyasa değeri ve aktif karlılığ 1 artırılırken net kâr marjı azaltılacaktır. Buna göre, Ryanair Holdings firmasının etkinlik skorunu 
değiştirmeyecek yeni girdi vektörü ne olmalıdır? Bu soruya cevap bulabilmek için Model (4)'ten yararlanılmıştır.

Ters VZA modelinin çözümü sonucunda Ryanair Holdings firmasının optimal girdi vektörünün $\alpha_{1}=(\approx 23308 ; 21,34)$ olması gerektiği hesaplanmıştır. $\mathrm{Bu}$ durumda, Ryanair Holdings firmasının mevcut girdi vektörü $x_{1}=(14853 ; 13,6)$ olduğuna göre, firma çalışan sayısını ve aktif toplamını \%56,92 oranında artırmalıdır. Ryanair Holdings firması için yukarıda gerçekleştirilen hesaplamalar Tablo 5'te gösterilmektedir.

Tablo 5: Ryanair Holdings Firması İçin Yeniden Yapılan Kaynak Tahsisi

\begin{tabular}{llll}
\hline Değişken & Orijinal değer & Yeni değer & $\%$ Değişim \\
\hline Piyasa değeri & 16,1 & 25 & $+\% 55,28$ \\
Aktif karlılı̆̆1 & 0,0956 & 0,15 & $+\% 56,92$ \\
Net kâr marj1 & 0,1429 & 0,1 & $-\% 30$ \\
Çalışan sayısı & 14853 & 23308 & $+\% 56,92$ \\
Aktif toplamı & 13,6 & 21,34 & $+\% 56,92$ \\
\hline
\end{tabular}

Çıktı yönelimli BCC-O modelinde etkinsiz çıkan $(\varphi=2.227)$ Türk Hava Yolları (THY) firmasının (F13) etkinlik skoru sabit kalmak şartıyla girdi vektörünün $x_{13}=$ (35205; 20,5)'ten $\alpha_{13}=(40000 ; \quad 15)$ şeklinde değiştirilmek istendiği varsayılmaktadır. Bu durumda THY firmasının yeni çıktı vektörü ne olmalıdır? Bu problemin optimal çözümü için Model (5)’ten yararlanılmıştır.

Ters VZA modelinin çözümü sonucunda THY firmasının optimal çıktı vektörünün $\beta_{13}=(7,799 ; 0,0427 ; 0,0628)$ şeklinde olması gerektiği hesaplanmıştır. $\mathrm{Bu}$ durumda, THY firmasının mevcut çıktı vektörü $y_{13}=(3,4 ; 0,0407 ; 0,064)$ olduğuna göre, firma etkinlik skorunu korumak istiyorsa piyasa değerini \%129,38 oranında, aktif karlılığını \%4,91 oranında artırırken, net kâr marjını ise \%1,88 oranında azaltmalıdır. THY firması için yukarıda gerçekleştirilen hesaplamalar Tablo 6'da gösterilmektedir.

Tablo 6: THY Firması İçin Yeniden Yapılan Kaynak Tahsisi

\begin{tabular}{llll}
\hline Değişken & Orijinal değer & Yeni değer & \%Değişim \\
\hline Çalışan sayısı & 35205 & 40000 & $+13,62$ \\
Aktif toplamı & 20,5 & 15 & $-26,83$ \\
Piyasa değeri & 3,4 & 7,799 & $+129,38$ \\
Aktif karl11ı̆̆ & 0,0407 & 0,0427 & $+4,91$ \\
Net kâr marj1 & 0,064 & 0,0628 & $-1,88$ \\
\hline
\end{tabular}

Diğer karar birimleri için de benzer hesaplamalar yapılabilir. 


\section{Sonuç}

Bu çalışmada Hadi-Vencheh ve Foroughi (2006) tarafından geliştirilen ters VZA modelleriyle Forbes dergisinin hazırladığ "Global 2000" listesindeki 21 adet havayolu firması için ters optimizasyon hesaplamaları yapılmıştır. Klasik VZA modelleri eldeki girdi ve çıktı değişkenlerini kullanarak karar birimlerinin etkinlik düzeylerini girdiye veya çıktıya yönelik olarak hesaplamaktadır. Bunun yanında, etkinsiz karar birimlerinin etkin duruma gelebilmeleri için girdi ve çıktılarında yapması gereken iyileștirme düzeyleri tespit edilmektedir. Ters VZA modelleri ise karar birimlerinin etkinlik skorları verili/sabit kalmak şartıyla girdi veya çıktılardaki miktar değişimleri karşısında diğer girdi/çıktılarda yapılması gereken değişim miktarlarını hesaplamaktadır.

Burada kullanılan yöntemin literatürdeki diğer ters optimizasyon modellerine karşı bazı üstünlükleri bulunmaktadır. Diğer ters VZA modelleri belirlenen bir karar biriminin bazı veya tüm çıktıları/girdileri artırıldığında girdilerin/çıktıların alması gereken yeni değerleri tahmin ederken, önerilen model eşzamanlı olarak çıktıların/girdilerin bazılarında artış bazılarında da azalma yapılması durumunda diğer girdilerde/çıktılarda yapılması gereken değişiklikleri hesaplayabilmektedir. Ayrıca sözkonusu model diğer ters VZA modellerinden farklı olarak ele alınan karar biriminin etkin olup olmadığından bağımsız olarak kaynak tahsisi yapmaktadır. Bunun yanında, önerilen model iyi yapılandırılmış ve sağlam bir teoriye sahip tek amaçlı veya ÇADP'ye dayalı bir modeldir.

Literatürde ters VZA modelleriyle etkinlik ölçümü yapılan az sayıda çalışma bulunması ve bu çalışma özelinde de havayolu firmalarının ele alındığı ilk girişimlerden biri olması nedeniyle bu çalışmanın literatüre katkı sunacağı düşünülmektedir. Uygulama sonuçları önerilen modelin havayolu firmaları ve diğer organizasyonlarda kaynakların yeniden tahsisi amaciyla kullanılabilecek pratik bir yöntem olduğu ve karar vericiler için sağlam ve yararlı bilgiler ürettiğini göstermektedir. Söz konusu model firma yöneticilerine kaynak tahsisi yanında, üretim planlama, performans ölçümü ve stratejik yönetim alanında yardımcı olabilecek bir modeldir. Girdi/çıktı yapılarını manipüle ederek karar vericilerin çeşitli üretim planları ve stratejilerinin etkinliğini analiz edebilmeleri mümkündür. 


\section{Kaynakça}

Abdollah, H., Ali, A., ve Majid, S. (2008). A DEA model for resource allocation. Economic Modelling, 25, 983-993.

Banker, R.D., Charnes, A., ve Cooper, W. W. (1984). Some models for estimating technical and scale inefficiencies in data envelopment analysis. Management Science, 30, 1078-1092.

Charnes, A., Cooper, W.W. ve Rhodes, E. (1978). Measuring the efficiency of decision making units, European Journal of Operations Research 2, 429444.

Chen, L., Wang, Y., Lai, F. J. ve Feng, F. (2017). An investment analysis for China's sustainable development based on inverse data envelopment analysis. Journal of Cleaner Production, 142(4), 1638-1649.

Emrouznejad, A. ve Yang, G.L. (2018). A survey and analysis of the first 40 years of scholarly literature in DEA: 1978-2016. Socio-Economic Planning Sciences, 61, 4-8.

Emrouznejad, A., Yang, G. ve Amin, G.R. (2019). A novel inverse DEA model with application to allocate the $\mathrm{CO} 2$ emissions quota to different regions in Chinese manufacturing industries, Journal of the Operational Research Society, 70(7), 1079-1090,

Farzipoor Saen, R. ve Seyedi Hosseini Nia, S. (2019), Evaluating after-sales service units by developing inverse network data envelopment analysis model, Benchmarking: An International Journal, DOI: 10.1108/BIJ-012019-0017.

Frija, A., Wossink, A., Buysse, J., Speelman, S. ve Huylenbroeck, G.V. (2011). Irrigation pricing policies and its impact on agricultural inputs demand in Tunisia: A DEA-based methodology. Journal of Environmental Management. 92(9), 2109-2118.

Ghiyasi, M. (2015). On inverse DEA model: the case of variable returns to scale. Computers \&Industrial Engineering, 87, 407-409.

Ghiyasi, M. (2017). Industrial sector environmental planning and energy efficiency of Iranian provinces. Journal of Cleaner Production 142(4), 328-2339.

Hadi-Vencheh, A., ve Foroughi, A.A. (2006). A generalized DEA model for inputs/outputs estimation. Mathematical and Computer Modelling 43, $447-457$. 
Hadi-Vencheh, A. Hatami-Marbini, A., Ghelej, B.Z. ve Gholami. K. (2014). An Inverse Optimization Model for Imprecise Data Envelopment Analysis. Optimization 64 (11), 1-14.

Jahanshahloo, G. R., Hosseinzadeh, L.F., Rostamy-Malkhalifeh, M. ve Ghobadi, S. (2014). Using enhanced russell model to solve inverse data envelopment analysis problems. The Scientific World Journal, 4, 1-10.

Lertworasirikul, S., Charnsethikul, P. ve Fang, S.C. (2011). Inverse Data Envelopment Analysis model to preserve relative efficiency values: the case of variable returns to scale." Computers and Industrial Engineering. $61,1017-1023$.

Lim, D.J. (2016). Inverse DEA with frontier changes for new product target setting. European Journal of Operational Research, 254(2), 510-516.

Steuer, R.E. (1986). Multiple Criteria Optimization Theory, Computation and Application. 2nd ed. Malabar, FL: Krieger.

Wegener, M. ve Amin, G.R. (2019). Minimizing greenhouse gas emissions using inverse DEA with an application in oil and gas. Expert Systems with Applications. 122, 369-375.

Wei, Q.L., Zhang, J. ve Zhang, X. (2000). An Inverse DEA Model for inputs/outputs estimate. European Journal of Operational Research 121, $151-163$.

Yan, H., We, Q. ve Hao. G. (2002). DEA models for resource reallocation and production input/output estimation. European Journal of Operational Research 136, 19-31.

Yu, A., Shao, Y., You, J., Wu, M. ve Xu, T. (2019). Estimations of operational efficiencies and potential income gains considering the credit risk for China's banks. Journal of the Operational Research Society, 70(12), 21532168 ,

Zhang, M. ve Cui. J.J. (2016). The extension and integration of the inverse DEA method, Journal of the Operational Research Society, 67(9), 1212-1220. 


\section{Resource Allocation in Global 2000 Companies using Inverse Data Envelopment Analysis}

$\overline{\text { Extended Abstract }}$

\section{Introduction}

An important challenge in the classical DEA models is how to maintain the efficiency score of a decision-making-unit against changes in the input-output set in the short term. In the literature, such problems are called resource re-allocation problems and considered as inverse optimization problems. The first inverse DEA (InDEA) model in the literature was developed by Wei, Zhang and Zhang (2000). The InDEA models calculate the amount of change in output (input) variables when the input (output) observations of a decision unit change provided that the efficiency score of that decision unit remains constant. Then, the InDEA problem is transformed and solved by a multi-objective linear programming model. After Wei et al. (2000), some extended and modified versions of this model have been proposed in the literature.

In this study, using the InDEA model introduced by Hadi-Vencheh and Foroughi (2006), an efficiency analysis was conducted for 21 airline companies included in the "Global 2000" list announced by the "Forbes" magazine.

\section{Method}

The "Forbes" magazine announces annually the "Global 2000" list which involves the best performing 2000 firms according to a weighted average of some selected metrics. In this study, an InDEA application using the IndEA method presented by Hadi-Vencheh and Foroughi (2006) was carried out for 21 airline companies that are included in the 2019 ranking of the "Global 2000" list.

Input and output variables used in the DEA application have been selected taking into consideration the relevant literature and data constraint. In the first stage of the application, efficiency measurement of the companies was carried out with classical CCR and BCC DEA models. Then, some reverse optimization calculations were employed for the efficient and inefficient units through the suggested InDEA model.

\section{Results and Discussion}

As per CCR-DEA model results, just Ryanair Holdings emerged as an efficient unit. The average efficiency score of the 21 airline companies is 0.444 , which can be considered as a low performance. According to input and output-oriented BCC DEA models, only five companies were found to be efficient. Ryanair Holdings is the only efficient unit as per three DEA models. Therefore, it can be concluded that airline companies did not use their resources efficiently in 2019.

In the second stage, a resource re-allocation problem was modeled and solved for Ryanair Holdings. The output vector of the company has been changed and the new optimal input vector has been calculated accordingly, on the condition that its efficiency score remained unchanged. Besides, similar resource allocation calculations have been conducted for the Turkish Airlines company, which is an inefficient unit as per the BCC-O model. The input vector of the company was altered and the new optimal output vector was computed such that its efficiency score maintained fixed. 


\section{Conclusion}

It is hoped that this study will contribute to the literature since it is one of the first attempts to deal with inverse optimization in the airline industry. The application results show that the proposed model is a practical method that can be used for the reallocation of resources in any homogenous organization as well as airline companies. The suggested model can help decision-makers for production planning, performance measurement and strategic management as well as resource allocation. By manipulating input/output structures, decision-makers can analyze the effectiveness of various production plans and strategies. 